\title{
Evaluation of Rating Scale Mental Effort (RSME) effectiveness for mental workload assessment in nurses
}

\author{
Ghanbary Sartang A, MSc ${ }^{1 *}$, Ashnagar M, MSc ${ }^{2}$, Habibi E, $\mathrm{PhD}^{3}$, Sadeghi S, MSc ${ }^{4}$ \\ 1- MSc of Occupational Health, School of Health, Isfahan University of Medical Sciences, Isfahan, Iran. 2- MSc of \\ Industrial Psychology, Bandar Abbas, Iran. 3- Professor, Dept. of Occupational Health Engineering, School of Health, \\ Isfahan University of Medical Sciences, Isfahan, Iran. 4- MSc of Industrial Engineering, Dept. of Industrial Engineering, \\ Ilam Branch, Islamic Azad University, Ilam, Iran
}

\begin{abstract}
Received: January 2017, Accepted: March 2017

Background: Mental workload is one of the factors that influence individuals' performance in the work environment as well as their efficiency and productivity. This study aimed to evaluate the effectiveness of the Rating Scale Mental Effort (RSME) for mental workload in nurses.

Materials and Methods: This study was descriptive and cross-sectional conducted on 75 nurses. For assessment of mental workload, first NASA task load index (NASA-TLX) and then RSME were measured. After from data gathering, the data were analyzed using SPSS, descriptive statistics, Student's independent t-test, analysis of variance (ANOVA) and Pearson correlation test.

Results: The results of the assessment workload using RSME and NASA-TLX index of nurses showed that nurses' workload was high. Pearson correlation test regression coefficient showed a good correlation between scores of RSME and NASA-TLX index $(r=0.72)\left(r^{2}=0.51\right)$. Also there was no significant association between age and work experience with mental workload score in RMSE $(\mathrm{P}=$ 0.09) $(\mathrm{P}=0.08)$ and NASA-TLX index $(\mathrm{P}=0.08)(\mathrm{P}=0.06)$. Student's t-test showed that no significant relationship was observed between sex and scores of RMSE $(P=0.08)$ and NASA-TLX index $(\mathrm{P}=0.07)$.

Conclusions: The results of this study showed that RMSE is a good method for assessing mental workload and there was acceptable correlation with NASA-TLX valid indices. RMSE index can be used to assess the mental workload.
\end{abstract}

Keywords: Workload, Effectiveness, Nurses

\section{Introduction}

Mental workload is a set of factors that affect the mental processing of information, providing decision making and individual reactions in the workplace. Workload has been reported to negatively affect the health of employees; long-term diseases may be a result of stress, monotony, mental dullness and fatigue at workplace. Moreover, excessive workload has been ranked first among the reasons for employee burnout, which has been commonly researched in recent years (1). Work-related stress and workload are two influential factors that threaten the fitness and health of a wide range of people in organizations. Over the past years, the subject of workload and its influence on organizations has received considerable attention in discussions related to organizational behavior. Mental workload is a set of factors that affect on the mental processes of information, providing decision making and individual reactions in the workplace. Workload and work-related stress affects individuals' safety, health, and comfort and have a direct relationship with people's performance $(1,2)$. Excessive workload has been identified as a significant stressor across different occupations. Jobs with high levels of workload and occupations within appropriate work

\footnotetext{
* Corresponding author: Aioob Ghanbary Sartang, School of Health, Isfahan University of Medical Sciences, Isfahan, Iran.

Email: aioobghanbary@ymail.com
} 
schedule would diminish operators' performance and results in memory impairments, irritability, and reduced learning capacity. Regulating task demands in a way that prevents individuals from being under load or overload has considerable importance to ensure their safety, health, comfort, and productivity (3). Allen and Mellor reported that nurses consistently recount that the quality of their work lives and work environment have deteriorated as a result of work content and work environment variables. Thirty to forty percent of the nurses surveyed reported a perceived decrease in quality of care over the past year as a result of the increasing workload environment demands (4). Work environment variables have been the least studied aspects of nursing workload. However, some studies have reported that work environment factors such as support by the manager and colleagues and work content have a stronger relationship with job satisfaction than economic variables. Nurses in five countries were surveyed over 20 months in 1998-1999 to determine factors related to nurses' job satisfaction and quality of care perceptions (5) Mental workload encompasses factors that impact the mental process of information processing, decision making, and individuals' response in the workplace. the subject of workload and its influence on organizations has received considerable attention in discussions related to organizational behavior (6). Measuring nurse's workload would have positive influences on the management of their workload and consequently on the provision of safety and quality of patients' care (7). Beckmann et al. investigated the problems associated with nursing staff shortage in intensive care units (ICUs) in Australia, and reported that nursing shortages would increase the rate of incidents and decrease the patients' safety and quality of care. Workload is a set of job requirements, the effort to perform the work, and efficiency and performance of individuals. Excessive workload leads to increasing mental and physical stress and job dissatisfaction and may be a threat to public health workers (8). Rating
Scale Mental Effort (RSME) has been developed for evaluation of workload. The RSME consists of a line with a length of 150 $\mathrm{mm}$ marked with nine anchor points, each accompanied by a descriptive label indicating a degree of effort and with the increasing, workload mental effort increase (9). The RSME is conceptually similar to the effort scale of the NASA-TLX; thus its use is also dependent on the ability and willingness of participants to interpret and report their state. The RSME scale requires no special device, the method is simple and cheap, quick response and applicability in the workplace without interfering with the work of individuals. According to the disadvantages of some indices of mental workload, this study aimed to evaluate the effectiveness of the RSME index for mental workload in nurses.

\section{Material and Methods}

The present study was cross-sectional, done in 2015 on 75 male and female nurses working in hospital in Ilam, Iran. Informed consent forms were signed by all individuals who participated voluntarily. Furthermore, the study was approved by the Ethics Committee. Inclusion criteria were no history of hypertension or other medical problems in the file of respondents and work experience of at least 6 months. Exclusion criteria were patients with pain or discomfort in various body organs, and the unwillingness of people to complete the questionnaire and the necessary cooperation. The data collection tools in this study were NASA-TLX questionnaire and RSME scale. The first part was the demographic information including age, gender and work experience. The second part was of the NASA-TLX questionnaire. NASA-TLX is one of the well-known subjective workload assessment tools, presented by Hart and Staveland. (10). the index consists of six subscales including mental demand, physical demand, temporal demand, performance, effort, and frustration. Reliability and validity of NASA-TLX 
questionnaire was confirmed by Mohammdi et al. and Cronbach's alpha for this scale was 0.89 (11). Third part was employing RSME scale that the score is 0 to 150 (Figure 1). RSME score is between 0 (absolutely no effort) and 150 (extreme effort) (9). The data were analyzed using SPSS (version 20.0, IBM Corporation, Armonk, NY, USA). Descriptive statistics, Student's independent t-test, analysis of variance (ANOVA) test and Pearson correlation test were used for statistical analysis. Ethical considerations in this study were observed. Respondents were also asked for the permission to review their ethical issue. Respondents were ensured that their information would remain anonymous and the data would be kept in a safe place and would not be used for any purposes other than for the present study. Also the value of $\mathrm{P}<0.05$ was considered statistically significant.

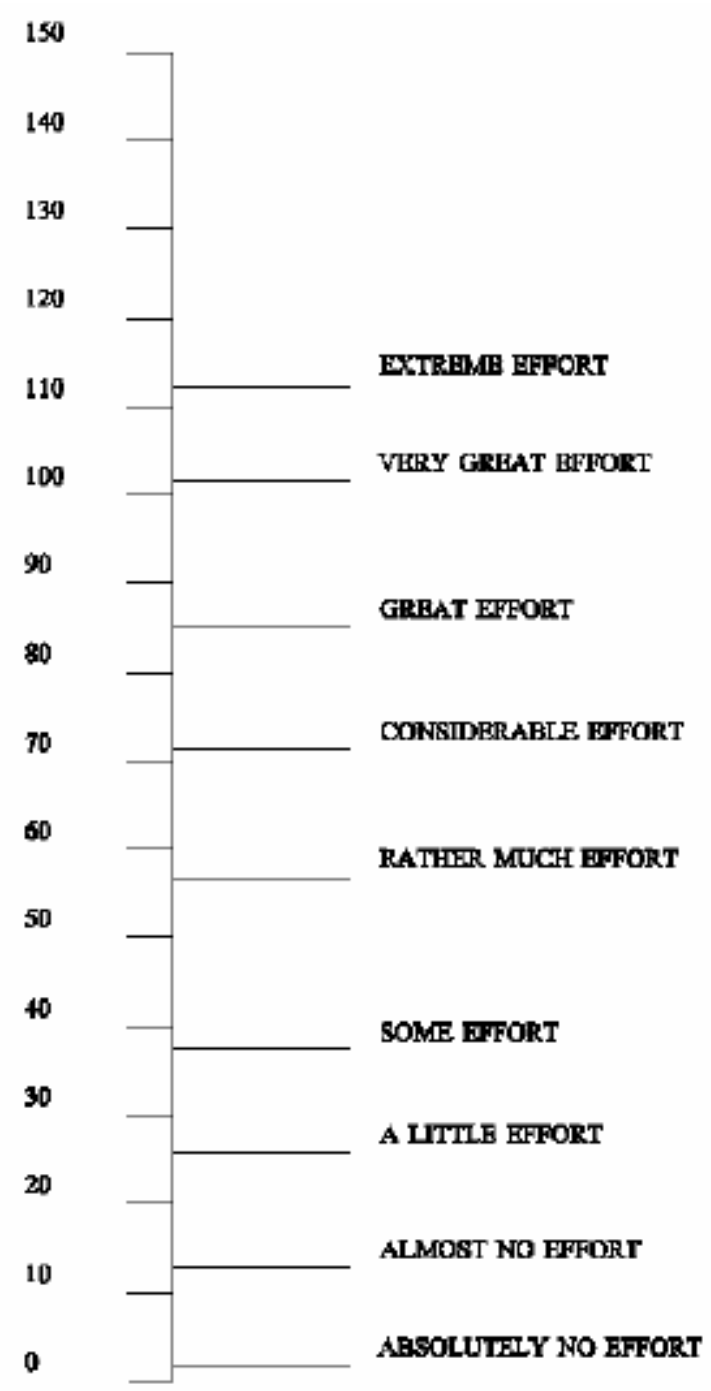

Figure 1: Rating Scale Mental Effort

\section{Results}

There were 75 participants in this study (34 males and 41 females). The mean [(standard deviation (SD)] age and work experience of the participants in this study are shown in table
1. The maximum and minimum ages of participants in this study were 45 and 24 years, respectively. The maximum and minimum work experiences of participants in this study were 22 and 2 years, respectively. 
Table 1: Mean (standard deviation) age, work experience participants

\begin{tabular}{cccc}
\hline Variable & Classification & Mean (SD) & Minimum-maximum \\
\hline \multirow{2}{*}{ Age } & Male & $33.10(5.17)$ & $26-45$ \\
\cline { 2 - 4 } & Female & $28.10(4.86)$ & $24-36$ \\
\hline \multirow{2}{*}{ Work experience } & Male & $16.20(4.28)$ & $4-22$ \\
\cline { 2 - 4 } & Female & $8.14(6.26)$ & $2-12$ \\
\hline
\end{tabular}

SD: Standard deviation

The mean (SD) Scores obtained for NASATLX and RSME scale presented in table 2. Based on the NASA-TLX index, mental demand had the highest score 85.1 (12.2) and physical demand had the lowest score (19.7)
45/59. Also score for RSME scale was 121.2 (39.6). Based on the NASA-TLX index (72.3) and RSME (121.2) scores, workload in nurses was high.

Table 2: Mean and standard deviation (SD) Scores obtained for NASA-TLX index and RSME scale

\begin{tabular}{ccc}
\hline Index & Mean & SD \\
\hline NASA-TLX & 59.5 & 19.1 \\
\hline Physical demand & 78.2 & 19.5 \\
\hline Temporal demand & 70.1 & 18.9 \\
\hline Performance demand & 61.1 & 23.3 \\
\hline Effort demand & 79.4 & 18.9 \\
\hline Frustration demand & 85.1 & 12.2 \\
\hline Mental demand & 72.3 & 18.6 \\
\hline Total score for NASA-TLX & 121.2 & 39.6
\end{tabular}

NASA-TLX: NASA task load index; RSME: Rating Scale Mental Effort

Pearson correlation test and regression coefficient showed a good correlation between RSME and total score from NASA-TLX index $(r=0.72)\left(r^{2}=0.51\right)$. Table 3 shows Pearson correlation coefficient and regression coefficient between RSME and each subscale of NASA-TLX index.
According to table 3, Pearson correlation test showed correlation between the RSME and each of the subscales of physical demand, temporal demand, performance, effort, and frustration median. Also there was a good correlation between RSME and mental demand subscale of NASA-TLX index.

Table 3: Pearson correlation coefficient and regression coefficient between RSME and each subscale of NASA-TLX index

\begin{tabular}{clccc}
\hline Subscale NASA-TLX index & RSME & $\begin{array}{c}\text { Pearson } \\
\text { correlation }\end{array}$ & $\begin{array}{c}\text { Regression } \\
\text { coefficient }\end{array}$ & P \\
\hline Physical demand & RSME & 0.56 & 0.32 & 0.03 \\
\hline Temporal demand & RSME & 0.65 & 0.42 & 0.02 \\
\hline Performance demand & RSME & 0.60 & 0.36 & 0.02 \\
\hline Effort demand & RSME & 0.53 & 0.28 & 0.04 \\
\hline Frustration demand & RSME & 0.66 & 0.43 & 0.02 \\
\hline Mental demand & RSME & 0.73 & 0.53 & 0.01 \\
\hline $\begin{array}{c}\text { Total score for NASA-TLX } \\
\text { index }\end{array}$ & RSME & 0.72 & 0.51 & 0.01 \\
\hline
\end{tabular}

NASA-TLX: NASA task load index; RSME: Rating Scale Mental Effort 
In this study, after Kolmogorov-Smirnov test and ensuring the normal distribution of variables, analysis of variance showed that there was no significant relationship between age and RSME $(P=0.09)$ and NASA-TLX index $(P=0.08)$. Also analysis of variance showed that there was no significant relationship between work experience with RSME $(\mathrm{P}=0.08)$ and NASA-TLX index $(\mathrm{P}=$ 0.06). The Student's independent t-test showed that there was no significant relationship between gender and RSME $(\mathrm{P}=0.08)$ and NASA-TLX index $(\mathrm{P}=0.07)$.

\section{Discussion}

One of the most important problems in nurses is a high nursing workload. A high workload may lead to increased human errors, compromised quality and safety of care, and reduced quality of working life. The purpose of this study was to evaluate the effectiveness of the RSME index for mental workload in nurses. Pearson correlation test showed a good correlation between RSME and total score of NASA-TLX index. Based on the NASA-TLX index and RSME, workload in nurses was high. Sarsangi et al. who conducted the evaluation of workload using NASA-TLX concluded that the NASA-TLX index is an appropriate method to evaluate the workload of nurses that is consistent with the results of this study (12). Widyanti et al. tested the validity and reliability of the RSME on students and teachers and concluded that the RSME is a suitable and fast method for evaluating mental workload that is consistent with the findings of the present study (13). Rafiee et al. assessed the mental workload in nurses and concluded that the NASA-TLX is a good index for the assessment of mental workload in ICU nurses (14). In this study, a good correlation was observed between the NASA-TLX index and RSME, which indicates that RSME is an appropriate method to assess mental workload. Malekpour et al. concluded there was no significant association between age and work experience with mental workload score for NASA-TLX findings of the present study confirms (15). Hoonakker et al. Concluded that nurses are exposed to high workload that is consistent with the findings of the present study (16). Richard et al. in their study concluded nurses in addition to the physical needs, are often exposed to other related factors including psychological needs, official disputes, pressure of time, lack of social support, conflicting needs, loss of work speed control (17). Rubio et al. who investigated workload indices concluded that self-reporting methods are good to assess mental workload (18). Bahadori et al. concluded that there was no relationship between age and gender and mental workload (19). Khandan et al. evaluated workload and job satisfaction and concluded that sex, age and work experience did not affect mental workload (20). Pearson correlation test showed correlation between the RSME and each of the subscales of physical demand, temporal demand, performance, effort, and frustration. Also there was a good correlation between RSME and mental demand subscale of NASA-TLX index. Ruiz-Rabelo et al. concluded that there were correlations between each subscale of NASA-TLX index, which was similar to the findings of this study (21). As work environments be-come more complex and new technologies are used by health care workers, the mental demand of these occupational groups is increased (22). Alizadeh et al. in their study concluded a high degree of nursing workload in ICU in three working shifts. Using NASA-TLX is recommended for determining the ratio of the nurses' activities to their numbers in order to provide high quality patient care in ICUs via allocating adequate number of nursing staff (23). Work-related stress and workload are two influential factors that threaten the fitness and health of a wide range of people in organizations. In another study, Yeung et al. found a linear correlation between mental demand and workload. Also, they showed a significant correlation between exhaustion due to long work hours and mental workload using 
the NASA-TLX (24). Analysis of variance showed that there was no significant relationship between work experience with RSME and NASA-TLX index. The Student's independent t-test showed that there was no significant relationship between gender in RSME and NASA-TLX index that matched with the results of the study by Malekpour et al. (15). The limitation of this study was the lack of opportunity to utilize a larger sample size.

\section{Conclusion}

The progress of technology and modern technology has changed today's workplaces and imposed more mental and cognitive demands to workers. Nurses are people who do important works in hospitals. Studies have shown that the strain level among nurses has inverse relation to the quality of care provided by them. The results of the present study showed that the RSME is a good index to evaluate the mental workload of nurses because of its acceptable correlation with NASA-TLX index. RSME is easy to use and fast as well as having a lower cost compared to other indices for evaluating the mental workload. Further studies on the working population with a larger sample size and different jobs should be performed for further evaluation of RSME scale.

\section{Acknowledgements}

The authors would like to thank all individuals who took part in this study, especially the participants. This study was funded and supported by the authors.

Conflict of interest: None declared.

\section{References}

1. Malekpour F, Mohammadian Y, Malekpour AR, Mohammadpour Y, Sheikh Ahmadi A, Shakarami A. Assessment of mental workload in nursing by using NASA-TLX. Journal of
Urmia Nursing and Midwifery Faculty 2014; 11(11):892-899.

2. Greenglass ER, Burke RJ, Moore KA. Reactions to increased workload: effects on professional efficacy of nurses. Applied Psychology 2003; 52(4):580-97.

3. Weinger MB, Englund CE. Ergonomic and human factors affecting anesthetic vigilance and monitoring performance in the operating room environment. Anesthesiology 1990; 73(5):995-1021.

4. Allen J, Mellor D. Work context, personal control and burnout amongst nurses. West $\mathrm{J}$ Nurs Res 2003; 24(8):905-17.

5. Aiken LH, Patrician PA. Measuring organizational traits of hospitals: the revised nursing work index. Nurs Res 2000; 49(3):14653.

6. Kiekkas P, Sakellaropoulos GC, Brokalaki H, Manolis E, Samios A, Skartsani C, et al. Association between nursing workload and mortality of intensive care unit patients. J Nurs Scholarsh 2008; 40(4):385-90.

7. Morris R, MacNeela P, Scott A, Treacy P, Hyde A. Reconsidering the conceptualization of nursing workload: literature review. J Adv Nurs 2007; 57(5):463-71.

8. Beckmann U, Baldwin I, Durie M, Morrison A, Shaw L.Problems associated with nursing staff shortage: an analysis of the first 3600 incident reports submitted to the Australian Incident Monitoring Study (AIMS-ICU). Anaesth Intensive Care 1998; 26(4):396-400.

9. Johnson A, Widyanti A. Cultural influences on the measurement of subjective mental workload. Ergonomics 2011; 54(6):509-18.

10. Hart SG, Staveland LE. Development of NASA-TLX (Task Load Index): results of empirical and theoretical research. In: Hancock PA, Meshkati N, edithors. Human mental workload. $1^{\text {st }}$ ed. Amsterdam, Netherlands: Elsevier; 1988. P.139-83.

11. Mohammadi M, Mazloumi A, Nasl Seraji J, Zeraati H. Designing questionnaire of assessing mental workload and determine its validity and reliability among ICUs nurses in one of the TUMS's hospitals. Journal of School of Public Health and Institute of Public Health Research 2013; 11(2):87-96.

12. Sarsangi V, Saberi HR, Hannani M, Honarjoo F, Salim Abadi M, Goroohi M, et al. Mental workload and its affected factors among nurses in Kashan province during 2014. Journal of Rafsanjan University of Medical Sciences 2015; 14(1):25-36.

13. Widyanti A, Johnson A, de Waard D. Adaptation of the Rating Scale Mental Effort 
(RSME) for use in indonesia. Int $\mathbf{J}$ Ind Ergon 2013; 43(1):70-6.

14. Rafiee N, Hajimaghsoudi M, Bahrami MA, Ghasemi N, Mazrooei M. Evaluation nurses' mental work load in emergency department: case study. Iranian Journal of Nursing Vision 2015; 3(4):43-50.

15. Malekpour F, Mehran G, Mohammadian Y, Mirzaee V, Malekpour AR. Assessment of mental workload in teachers of Hashtrud city using NASA-TLX mental workload index. Pajoohandeh 2014; 19(3):157-61.

16. Hoonakker P, Carayon P, Gurses A, Brown R, McGuire K, Khunlertkit A, et al. Measuring workload of ICU nurses with a questionnaire survey: the NASA task load index (TLX). IIE Trans Healthc Syst Eng 2011; 1(2):131-43.

17. Richard JH, Samuel JA, Kamisha HE, Rainu $K$, Kathleen $M$, Neal $P$, et al. Nursing workload and its effect on patient and employee safety. Proc Hum Factors Ergon Soc Annu Meet 2007; 51(11):760-4.

18. Rubio S, Díaz E, Martín J, Puente JM. Evaluation of subjective mental workload: a comparison of SWAT, NASA-TLX, and workload profile methods. Applied Psychology 2004; 53(1):61-86.

19. Bahadori M, Ravangard R, Raadabadi M, Mosavi SM, Fesharaki MGh, Mehrabian F. Factors affecting intensive care units nursing workload. Iran Red Crescent Med J 2014; 16(8):e20072.

20. Khandan M, Roshan Zamir S, Maghsoudipour M. Survey of workload and job satisfaction relationship in a Productive company. Iran Occupational Health Journal 2012; 9(1):30-6.

21. Ruiz-Rabelo JF, Navarro-Rodriguez E, DiStasi LL, Diaz-Jimenez N, Cabrera-Bermon J, Diaz-Iglesias C, et al. Validation of the NASATLX Score in ongoing assessment of mental workload during a laparoscopic learning curve in bariatric surgery. Obes Surg 2015; 25(12):2451-6.

22. Wilson MR, Poolton JM, Malhotra N, Ngo K, Bright E, Masters RS. Development and validation of a surgical workload measure: the surgery task load index (SURG-TLX). World J Surg 2011; 35(9):1961-9.

23. Alizadeh M, Heidari Gorji MA, Khalilian AR, Esmaeili R. Assessment of nursing workload and related factors in intensive care units using the nursing activities score. Journal of Mazandaran University of Medical Sciences 2015; 24(122):147-57.

24. Yeung SS, Genaidy A, Deddens J, Sauter S. The relationship between protective and risk characteristics of acting and experienced workload, and musculoskeletal disorder cases among nurses. J Safety Res 2005; 36(1):85-95. 